\title{
Introductory Teaching Tool Utilizing Immunohistochemistry to Explain the Placenta
}

"The womb may be more important than the home"

J.P. Barker

\author{
Mercedes Olaya-C and Jorge Andrés Franco \\ Department of Pathology, The Medical School, Pontificia Universidad Javeriana-San Ignacio University Hospital, Bogota, Colombia
}

${ }^{\star}$ Correspondence to: Mercedes Olaya-C, Department of Pathology, The Medical School, Pontificia Universidad Javeriana-San Ignacio University Hospital, Bogota, Colombia; E-mail: olaya.m@javeriana.edu.co

Received: May 11, 2018; Accepted: May 22, 2018; Published: May 24, 2018;

\begin{abstract}
Introduction: The placenta occupies an important place in science, medicine, and law; yet, it remains a difficult organ to explain and understand because of its unique characteristics.
\end{abstract}

Methods and Materials: A descriptive observational study was carried out on placentas. Placental components were illustrated with diagrams and corresponding microphotographs were highlighted by immunohistochemical staining to identify cell-types and structures.

Results: The placenta was diagrammatically demonstrated by dividing it into chorionic plate with umbilical cord, basal plate and placental disc. In highlighting the histological components, approximately 80 immunohistochemical preparations were taken into account

Conclusion: Placental complexity continues to be the critical interface for maternal-fetal dialogue. Better educational methods are needed to explain the complex pathophysiology of this essential organ. Improving placental education is the key to attracting young researchers dedicated to this field.

Key words: Histology; immunohistochemistry; medical education; placenta; trophoblast.

\section{Introduction}

The placenta is a complex and misunderstood organ not only because it is exclusively vascular in nature and possesses allograft-like characteristics, but also because it ceases to function at week 40 of gestation. The placenta is temporary because it is unable to be efficient after reaching 40 weeks of gestation; it is no longer able to sustain the rapidly growing fetus. Its vascular features are unique because the placenta manages two individual circulatory systems belonging to two different people (mother and fetus). It is allograft-like because it comes from two individuals with unique genotypes from the same species. .

The placenta has been described as "the most important organ of the body, but paradoxically the most poorly understood" $[1,2]$. Examination of the placenta by the pathologist has been defined as a "gestation diary" [3]identifying conditions that are likely to recur in subsequent pregnancies, separating clinical syndromes into distinct pathological phenotypes for further investigation, and uncovering the underlying cause of unexpected adverse outcomes. Classification of placental lesions has evolved from being a purely descriptive exercise through a stage in which the major pathophysiological processes such as disorders of maternal implantation and the amniotic fluid infection syndrome were first described to a recently proposed comprehensive classification system that includes all of the major maternal and fetal vascular and infectious and idiopathic/immune inflammatory processes (Amsterdam Placental Workshop Group; assessment of stillbirth could not be complete without the study of the placenta and vice versa [4]. Therefore, in medical-legal cases, "the placenta has played a pivotal role, and at times, stood in the forefront, not only as participant but also as witness" [5]. These types of cases have recently increased, in which the placenta is like a "black box" in a trial involving a medical malpractice lawsuit [6].

Odd as it may seem, study of the placenta in pathology departments is not mandatory, on the premise that most babies will be born healthy and therefore the placenta must also be healthy [7]. Most of the organs or pieces of tissue removed from any patient must be sent to the pathology lab for examination; the placenta is the most common exception to this requirement. Incongruous, as well, is the fact that the placenta is currently one of the most frequently examined specimens in pathology departments due to the large number of deliveries. The importance of the placenta is now a recognized factor in newborn prognosis, essential to explaining the causes for stillbirth; and, in the long-term it acquires medico-legal aspects when later childhood neurodevelopmental delay are diagnosed $[8,9]$.

Additionally, the importance of placental examination in clarifying failed maternal reproduction has been on the rise in recent decades. Placental examination can also be directly requested by families themselves who want to know how etiology, prognosis, future outcomes, probabilities and any other information could be useful in analyzing future gestations among their own relatives. Therefore, in 
order to obtain the greatest amount of answers for families, the joint interpretation of the binomial fetus and placenta is mandatory $[10,11]$. Furthermore, science appears to be rediscovering the placenta. The trophoblast and the other placental components are now being widely researched [12]. New techniques in molecular biology have brought about significant advances in epigenetics, imprinting, cell culture, genetic diseases, complete genome sequencing, and immunology, all of which have helped to expand our knowledge about placental components and their functions. Diseases like preeclampsia, with its enormous impact on fetal and maternal morbidity and mortality, are now being focused on, but despite the existence of a multitude of theories $[13,14]$, benefits to clinical application have yet to be seen. We still do not know enough about interactions between trophoblast and maternal immune cells [histiocytes and lymphocytes], the role of trophoblast in decidual and spiral arterial invasion, and other related issues.

- The concept of two different circulatory systems belonging to two different individuals in the same organ is unique.

- Understanding how each joined individual pumps its own circulation in not easy, even among students in advanced specialized medical studies.

- The importance of the umbilical cord [UC] as a structure that carries fetal blood, which is akin to having the aorta and the cava vein outside the body, is not sufficiently recognized [Figure 1 highlights the placental tree as a continuity of fetal circulation].

- The presence of a variety of special, temporary and unique cells -the trophoblast- with tumor-like behavior and tumor-like appearance [invasive capacity, anisonucleosis, nucleomegaly, nuclear hyperchromasia and pleomorphic nuclei] [9], whose subdivisions in appearance and shape can be difficult to understand and even to pronounce [cytotrophoblast, syncytiotrophoblast, endovascular extravillous trophoblast, interstitial extravillous trophoblast].

- The placenta does not respond in a single way to injuries, for example, to hypoxia [10]297 with uterine type of chronic hypoxic placental injury (group 2

- The variety of normal and abnormal aspects that depend upon the age of gestation, its maturation [19]: nucleated fetal red blood cells [20], fibrin, syncytial knots, multinucleated extravillous trophoblast, amount of spiral artery muscle [21] and for this purpose they are remodelled into highly dilated vessels by the action of invading trophoblast (physiological change.

- The placenta is an organ that plays many roles; no organ can match the placenta for functional diversity [1].

The principal aim of this article was to propose a clear simple method to describe the placenta to those individuals who have had little or no previous exposure; additionally, some specific aspects have been emphasized so that the significance, continuity and wholeness of the fetal-placental unit may be completely understood.

\section{Methods And Materials}

We performed a descriptive observational study on the placenta and used immunohistochemical staining to highlight its components; diagrams and microphotographs were referred to when explaining placental structures and functions. Placental cases were collected that corresponded to samples studied at the PUJ-HUSI Department of Pathology from 2007 to 2017. A diagram of the placenta which illustrated its components, was created. This was accompanied by corresponding microphotographs, highlighted by immunohistochemistry staining, which differentiated cells and structures. Placental components were divided into fetal and maternal directions and vice versa, following the tree [Figure 1]. Then, slides were chosen from the available cases that had been collected for both diagnosis and research, and that had been tested with conventional immunohistochemistry on paraffin embedded tissue [BAX, FAS, bcl2, p57, cMyc, IGF2, FGF2, VEGFA, VEGF R1, MMP1, VEGFB, TGFB3, Ki67, PLGF, thrombomoduline]. Antibodies that best highlight specific placental cell types were chosen for this purpose. Microphotographs were taken and included with explanatory diagrams.

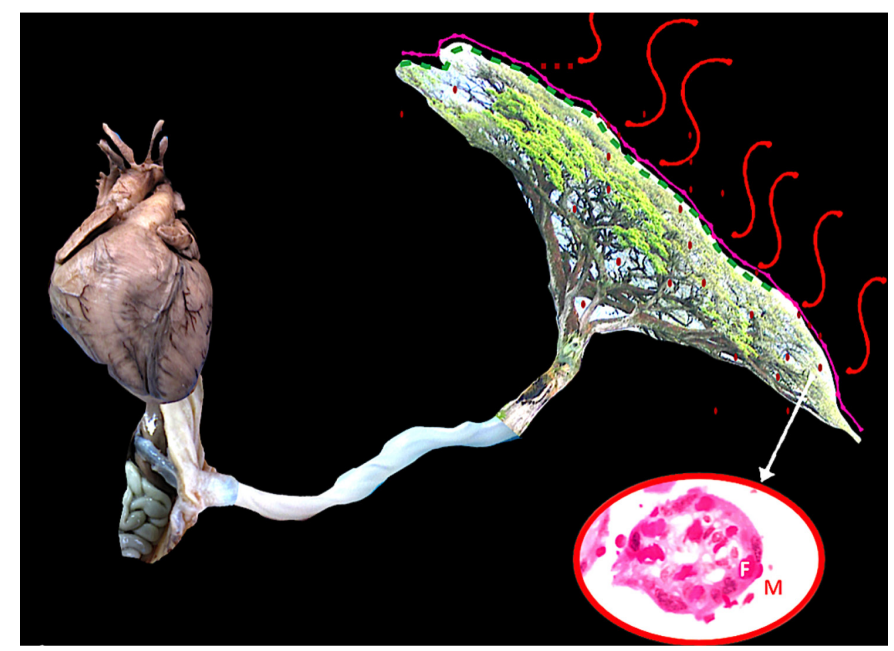

Figure 1. Unit fetus, umbilical cord and placenta

The figure represents the continuity from the fetal heart to the distal villi in the placenta. Blood is pumped from the fetal heart through the entire fetal body which also needs to be impulsed outside the body and pass through the umbilical cord, as long as it can be. When the blood reaches the chorionic plate in the placenta, it is distributed in many branches, up to the last villi where the capillaries are close to the mother's blood: her blood functions like air moving around leaves. Afterwards, oxygenated blood must return to the fetal heart.

In the close up, a villous is shown. Fetal capillaries are carrying fetal circulation (F); around it, maternal blood is present (M).

\section{Results}

An overall diagram of the placenta was created [Figure 2] which was subsequently divided into the chorionic plate with umbilical cord, basal plate and placental disc. Ordinary placentas and placentas previously chosen for other studies were collected to illustrate structures, especially those that have received scant recognition. A work checklist was created and filled with data for future use with this collection; microphotographs were taken and selected for the abovementioned purpose. Approximately 80 immunohistochemical preparations were taken into account when spotlighting histological components. Some of said antibodies have not been described in the placenta. Different trophoblast cell types exhibit different expressions; for example, villous syncytiotrophoblast does not have the same 
expression as subchorionic plate syncytiotrophoblast or basal plate syncytiotrophoblast, for the MMP1 antibody, the explanation of which is beyond this paper's goal.

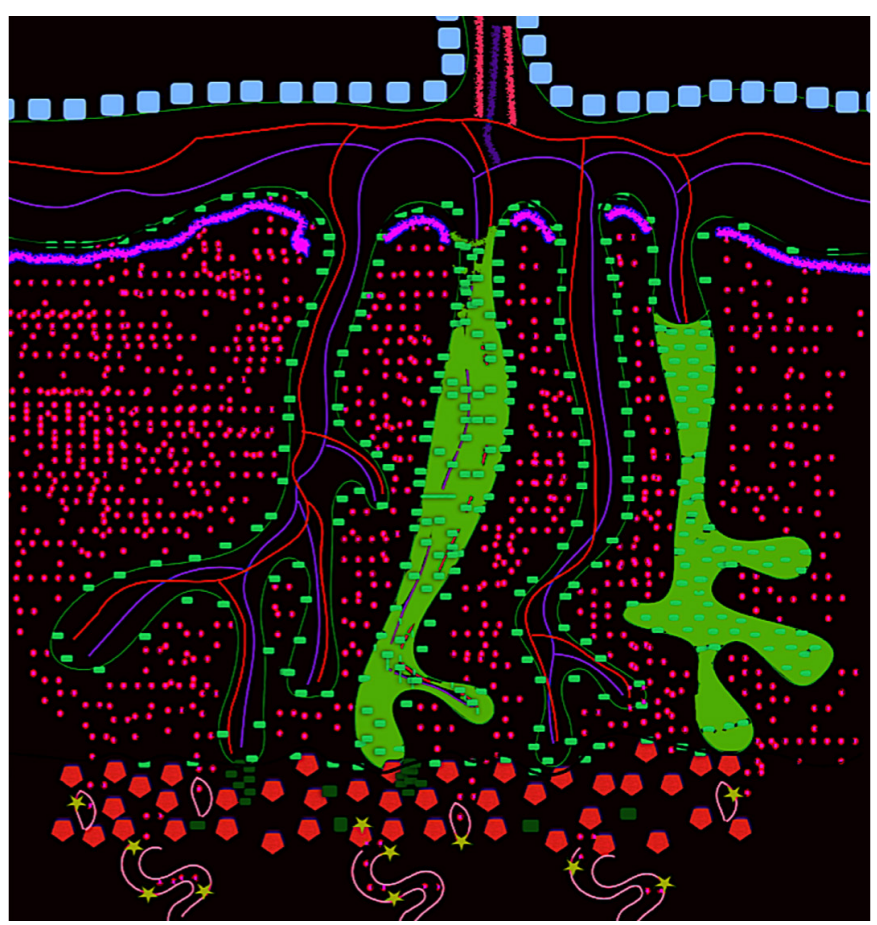

Figure 2. The placenta

The figure represents the placenta. Spiral arteries (A) are shown in the basal plate. Decidual cells (red hexagons) are mixed with endovascular extravillous trophoblasts (light green stars), interstitial extravillous trophoblasts (dark green rectangles); these cells also form columns of trophoblasts (B)

The trophoblast is always green in this figure, regardless of type. Syncytiotrophoblast overcoats the whole placental tree (light green rectangles) (D) and completely separates both circulatory systems (maternal and fetal); syncytiotrophoblast is also seen in maternal and fetal borders. Cytotrophoblast is beneath the villous syncytiotrophoblast. Fibrin is represented by a pink line $(\mathrm{C})$. The amnion is on the fetal side (gray rectangles). In the chorionic plate, arterial (E) and venous vessels (F) can be seen, connected to the UC (G).

However, we suggest that cells of the same origin and denomination could have very different functions, but these have not been studied in-depth nor are they well understood. Simultaneously, the expressions of some antibodies were surprisingly high; as was the case of FAS, with regards to apoptosis; others were surprisingly low, like bcl2, with regards to anti-apoptosis. Protein expression analysis via immnunohistochemistry shows that cells normally seen as equals, are not; furthermore, their activities include unexpected biochemical pathways. The best antibodies chosen to be photographed and described are listed in Table 1.

\section{Discussion}

The complexity of the placenta can be simplified by following the blood flow through the fetal extracorporeal circulation from the umbilical cord, where the umbilical vein and arteries are crucial for fetal life, and on toward the maternal bed with its remodeled spiral arteries. Maternal arteries and their abnormal remodeling are now recognized as linked to some of the most important adverse outcomes. In order to better understand the placenta, it should be divided into the:
- Fetal surface-umbilical cord

- Fetal surface-chorionic plate

- Placental disc

- Maternal surface-basal plate

\section{Fetal surface- umbilical cord}

The placenta is an extension of the fetal cardiovascular system [Figure 1]. The umbilical cord [UC] is the bridge between the intra and the extracorporeal circulations. The UC protects the whole fetal blood volume by means of coiling and with Wharton's jelly. Starting from the outside, the first layer of the UC is the amnion, which is firmly attached to the cord that protects Wharton's jelly [Figure 3D]. Beneath the amniotic epithelial cells, Wharton's jelly is interspersed with stromal cells [Figures 3C and 3D]; these are few, and they are spread haphazardly between the amnion and the blood vessels. The vessels are surrounded by myofibroblast, which are inconspicuous. The umbilical arteries and the vein lack a real adventitia, which is expected because of their size; therefore, the muscular layer is interlaced with Wharton's jelly structures [Figure 3B]. This is why the umbilical vessels cannot be dissected. The venous and arterial walls contain smooth muscle [Figure 3B], perivascular and endothelial cells [Figure 3B]. The vein also has an unusual subintimal elastic layer [23]. The umbilical arteries, on the contrary, do not have an inner elastic layer as do the other great arteries within the body [24]porous Wharton's jelly, two umbilical arteries, and one umbilical vein, are designed to protect blood flow to the fetus during a term pregnancy. The outer amnion layer may regulate fluid pressure within the umbilical cord. The porous, fluid filled Wharton's jelly likely acts to prevent compression of the vessels. Blood flow is regulated by smooth muscle surrounding the arteries that is intermingled with a collagen based extracellular matrix (ECM; furthermore, in spite of their caliber, UC arteries have a much thinner outer elastic layer than would be expected [23]. The venous diameter is double that of the arterial diameter [Figures 4B] [25].

Once the UC contacts the placental disc on the fetal surface, ideally on the center or close to it and at a 90-degree angle, the chorionic plate has been reached [Figure $4 \mathrm{~A}$ ].

\section{Fetal surface- chorionic plate}

To reiterate, the closest layer to the fetus is the amnion [Figures $4 \mathrm{C}, \mathrm{E}$ and $\mathrm{F}]$, but this amnion is not firmly attached here, as it is on the cord; conversely, the union is virtual. Grossly the chorionic plate is bluish in color, but up close, transparent, which allows the vessels branching out on the surface to be seen. It should also be possible to distinguish veins from arteries [Figure 4A]: the arteries are on top of the veins. Histologically, the amnion looks like the UC, with a single organized layer of cuboidal cells.

Located under the amnion is the chorionic tissue [Figures $4 \mathrm{D}$, E and F]; it also looks like the UC: hypocellular and with large fetal vessels. Each fetal vessel should not be more than three times larger than the adjacent vessels [24]such as true knots (TK. Behind the chorionic layer the trophoblastic cell layer is found [Figures $4 \mathrm{E}$ and $\mathrm{F}$ [green]], the trophoblast cell layer covers the inner part of the placenta including each small terminal villi [25], which has also been described 
in mice [26]. The human placenta is hemochorial, the maternal blood has contact with the trophoblast, but the trophoblast isolates the branched tree, including the chorionic and basal plates inside the disc [Figures 2, 4, 5 and 6].

Table 1. Antibodies in Figures:

[]: Concentration of primary antibody; C: clonality, M: monoclonal, P: polyclonal.

\begin{tabular}{|c|c|c|c|c|c|c|}
\hline FIG & ANTIBODIES & CODE & LAB & [l] & C & Control \\
\hline Fig 3 & $\mathrm{cMyc}$ & (9E11): sc-47694 & Santa Cruz Biotechnology Inc & $1: 1,000$ & M & Colon \\
\hline Fig 3 & $\mathrm{cMyc}$ & (9E11): sc-47694 & Santa Cruz Biotechnology Inc & $1: 1,000$ & M & Colon \\
\hline Fig 3 & $\mathrm{cMyc}$ & (9E11): sc-47694 & Santa Cruz Biotechnology Inc & $1: 1,000$ & M & Colon \\
\hline Fig 3 & FAS & $(C-20): s c-715$ & Santa Cruz Biotechnology Inc & 01:25 & $\mathrm{P}$ & Liver \\
\hline Fig 4 & VEGF-A & GTX102643 & GeneTex & & $\mathrm{P}$ & Riñón \\
\hline Fig 4 & VEGF-R1 & GTX15294 & GeneTex & & $\mathrm{P}$ & Placenta \\
\hline Fig 5 & VEGF-R1 & GTX15294 & GeneTex & & $\mathrm{P}$ & Placenta \\
\hline Fig 5 & p 57 & SPM308:sc-56456 & Santa Cruz Biotechnology Inc & 01:50 & M & Placenta \\
\hline Fig 5 & VEGF-A & GTX102643 & GeneTex & & $\mathrm{P}$ & Riñón \\
\hline Fig 6 & MMP1 & GTX100534 & GeneTex & & $\mathrm{P}$ & $\begin{array}{l}\text { Endometrial } \\
\text { carcinoma }\end{array}$ \\
\hline Fig 6 & VEGF-R1 & GTX15294 & GeneTex & & $\mathrm{P}$ & Placenta \\
\hline Fig 6 & VEGF-B & $(\mathrm{J}-14 \mathrm{I}): \mathrm{sc}-80442$ & Santa Cruz Biotechnology Inc & $01: 50$ & M & Placenta /Heart \\
\hline Fig 7 & VEGF-R1 & GTX15294 & GeneTex & & $\mathrm{P}$ & Placenta \\
\hline
\end{tabular}

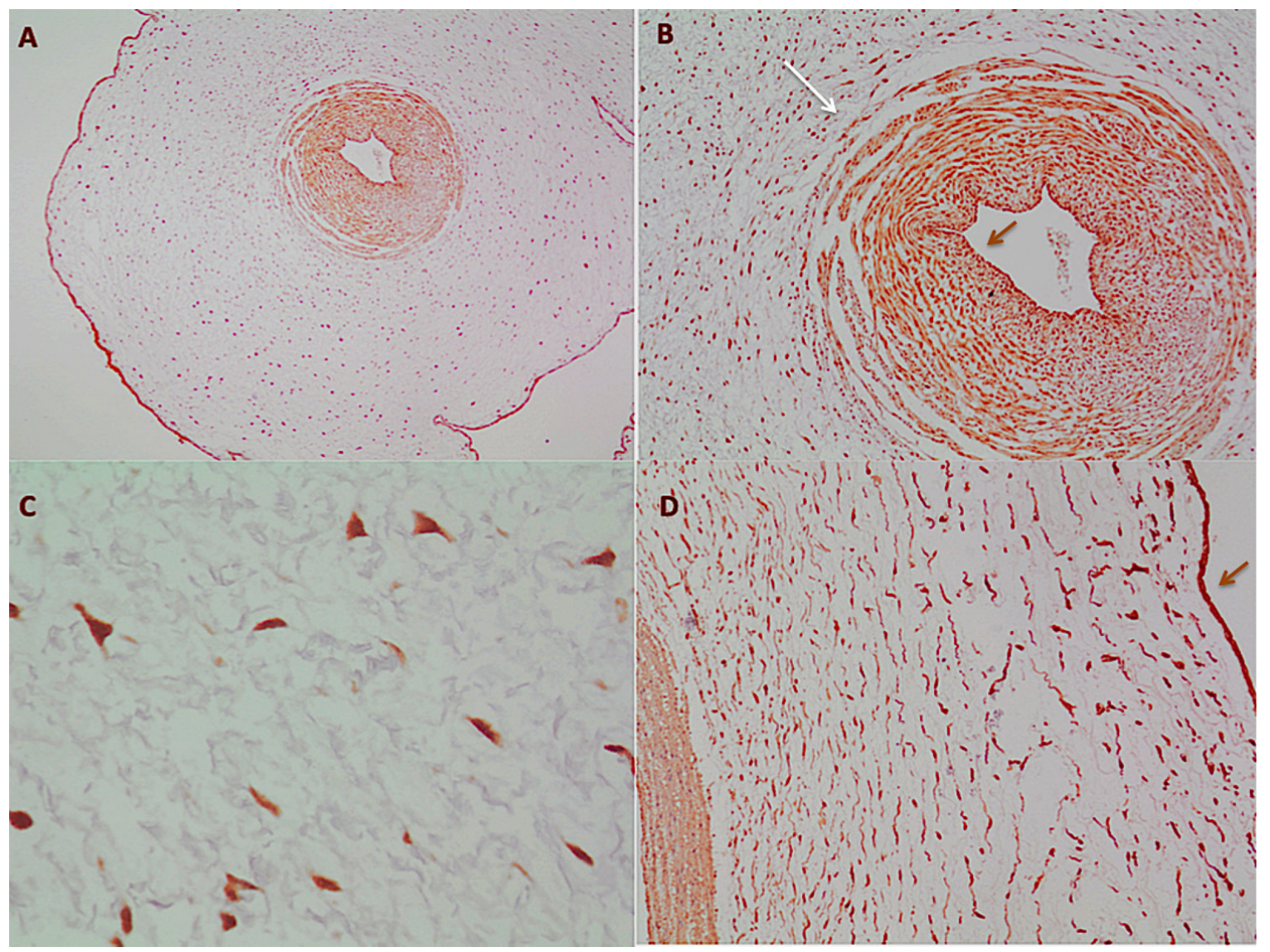

Figure 3. The umbilical cord

A- The UC clearly exhibits the amnion surrounding it; in the center, the umbilical artery (cMyc 4x). B- In the umbilical artery, the smooth muscle with the nondefined border (long white arrow) can be seen; in the center of the vessel, darker cells, the endothelium (short brown arrow) (cMyc 10x). C- Stromal cells in the Wharton's Jelly (cMyc 40x). D- From left to right, vascular wall, Wharton's Jelly with stromal cells and in the border, the amnion (brown arrow) (FAS 10X). 


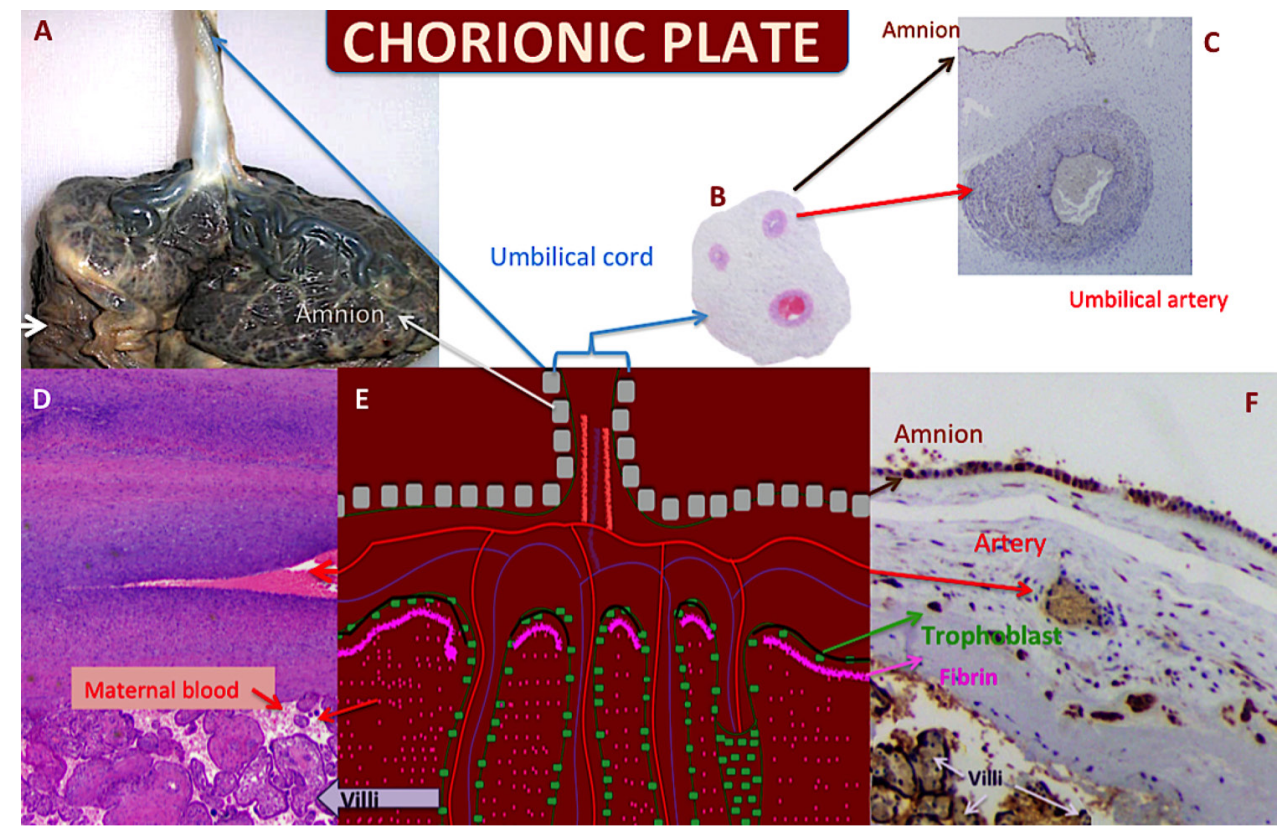

Figure 4. The chorionic plate

A- Gross placental morphology on the fetal side. UC Insertion is close to the center and placed at a $90^{\circ}$ angle; the placenta is covered by the amnion; fetal surface is bluish in color. The chorionic vessels can be seen on the surface. The membranes are upside down (white arrow). B- A histological microphotograph of the UC with two arteries and one vein; the vein is twice the size of the arteries. C- The amnion covering the UC surface can be seen at the top and the artery exhibits its thick smooth muscular layer (VEGF-A 2x). D- The chorionic vessel is shown and the villi are immediately below it (H\&E 2X). E- The figure represents the chorionic plate and its continuity with the UC and with the villi. F- As in Figure D, the amnion can be recognized at the top, the artery is in the chorionic plate. Here, the fibrin is clear and the syncytiotrophoblast is highlighted (green arrow) (VEGF-R1 10X); this syncytiotrophoblast is covering the chorionic plate, isolating the maternal and fetal circulatory systems.

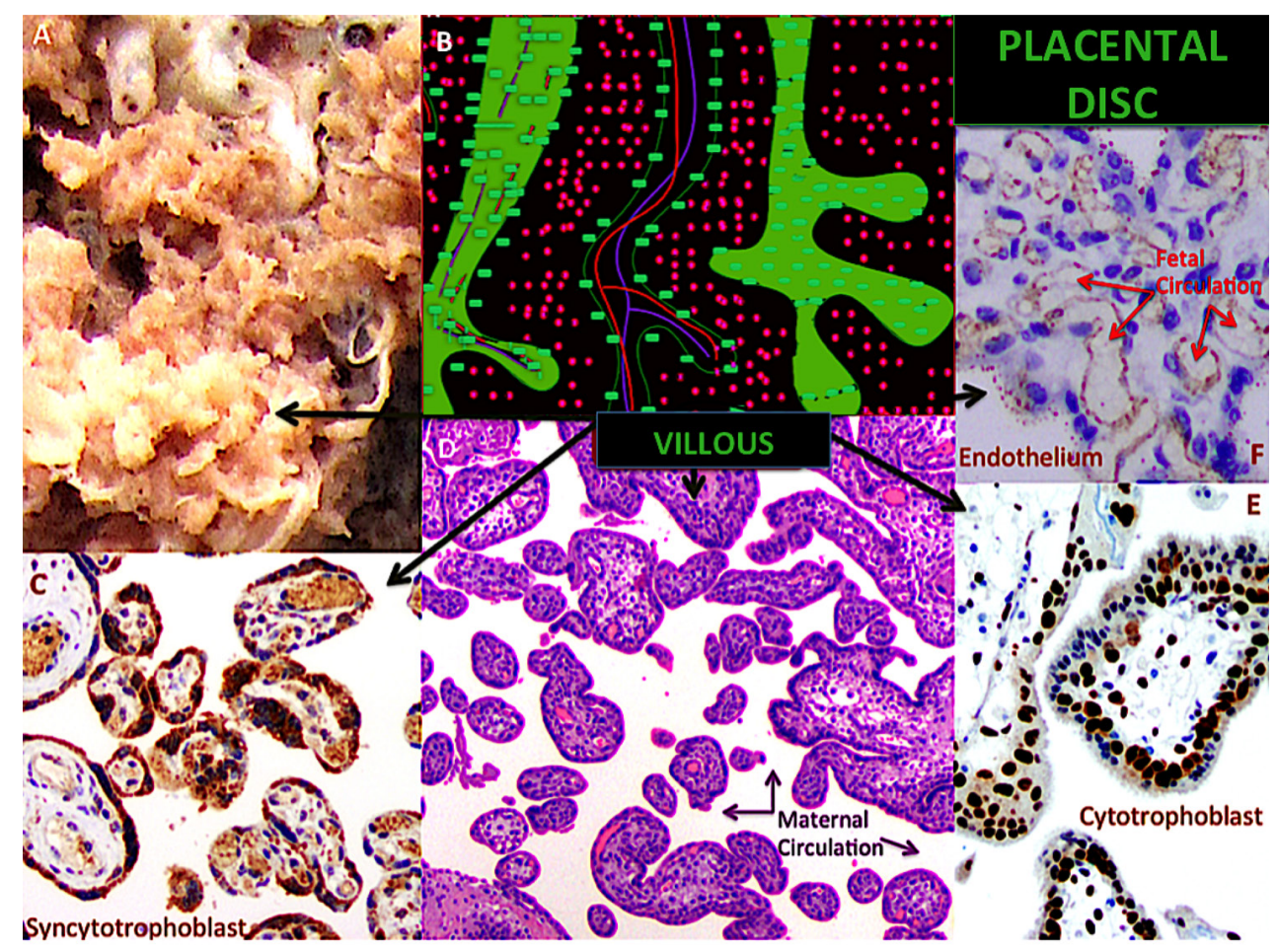

Figure 5. The placental disc

A-Gross placental morphology of the villi, which resemble tiny fingers. B- The figure represents the inside of the placenta; each villous carries capillaries and is covered by syncytiotrophoblast. C-Transversal view of the villous placental tree; the syncytiotrophoblast is highlighted; note the syncytial knots (VEGF-R1 20X). D- Transversal view of the villous placental tree; villi are separated; in the space between them, maternal blood circulates (H\&E 10X). E- Transversal view of the villous placental tree; the cytotrophoblast is highlighted, behind the syncytiotrophoblast (p57 10X). F- Transversal view of the villous placental tree; fetal capillaries are highlighted insight the villi. The surrounding blue colored nucleuses correspond to stromal cells (VEGF-A 20X). 


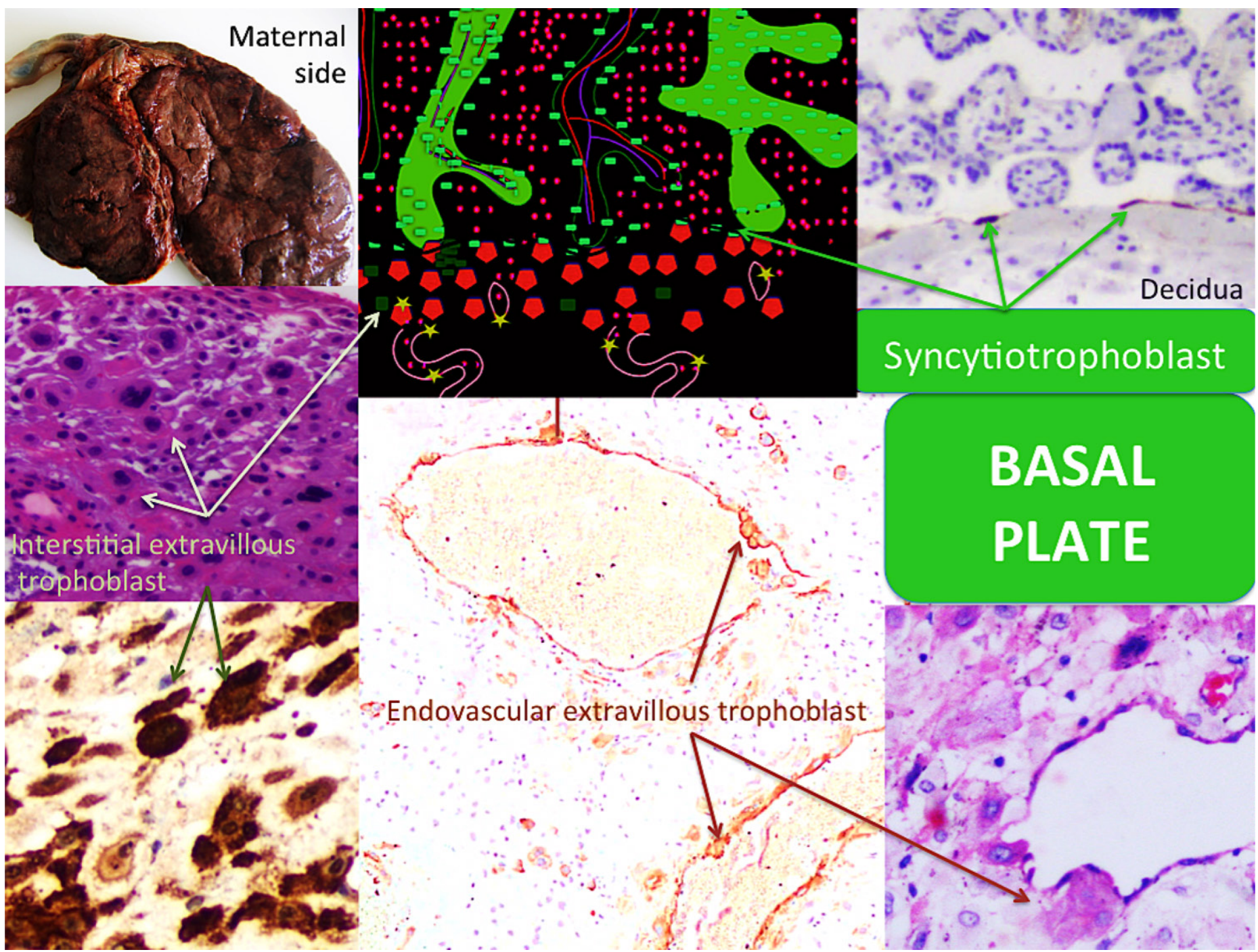

Figure 6. The basal plate 1

A- Gross placental morphology of maternal surface; the placental cotyledons are discreetly outlined. Membranes were removed. The UC is seen on top. B- The figure represents some contact between the anchoring villi and the basal plate. The syncytiotrophoblast continues isolating both circulations, now covering behind the decidua (C), like a line. Also, the trophoblast columns can be distinguished (dark green). The interstitial extravillous trophoblast (dark green squares) and intravascular extravillous trophoblast (light green stars) in spiral artery walls are represented. C- The syncytiotrophoblast behind the basal plate is seen (MMP1 20X). D- The basal plate with its mixed cell population is exposed: decidualized (maternal) cells and interstitial extravillous trophoblast (arrows) (H\&E 10X). E-Immunohistochemistry microphotograph highlights the interstitial extravillous trophoblast (green arrows) (VEGF-R1 10X). F- Immunohistochemistry staining highlights the endovascular extravillous trophoblast (VEGF-B 10X). G- The endovascular extravillous trophoblast can be easily seen in routine preparations (H\&E 40X).

\section{Placental disc}

Trophoblast cells that protect the contact area, which extends all around the internal placental disc, between the two circulations, were highlighted in Figure 2 [light green rectangles]. Figures 2 to 6 show this trophoblast cell layer, and demonstrate their different roles in different locations since their respective protein expression is not the same. The placental disc can be represented as a villous tree, whose branches only distribute fetal necessities, moving from the fetal heart to the terminal villi upstream and downstream. The maternal blood supply in the intervillous spaces is distributed like air among branches and leaves [27], coming from remodeled arteries which have lost their muscle and have larger lumens and are less resistant to flow, and, hence, have less pressure [Figures 1, 2, 5 and 7]. The intervillous space should only contain maternal blood, without obstructions [Figures 5]. Syncytiotrophoblast cells are multinucleated fused cells whose surfaces are covered by a dense network of branched microvilli that encompass an area of $12 \mathrm{~m}^{2}$ in the final stage of human gestation [28] [Figure 5].

Fetal and maternal circulations are separated. The fetal system is located in the villi and is separated from the maternal system by: the vasculosyncytial membrane;(this membrane decreases in thickness as pregnancy progresses; it is composed of: the endothelium inside the villi, the basement membrane and some connective tissue), by a layer of discontinuous cytotrophoblast cells that also decreases progressively, and by the syncytiotrophoblast and its basal membrane [29]. 


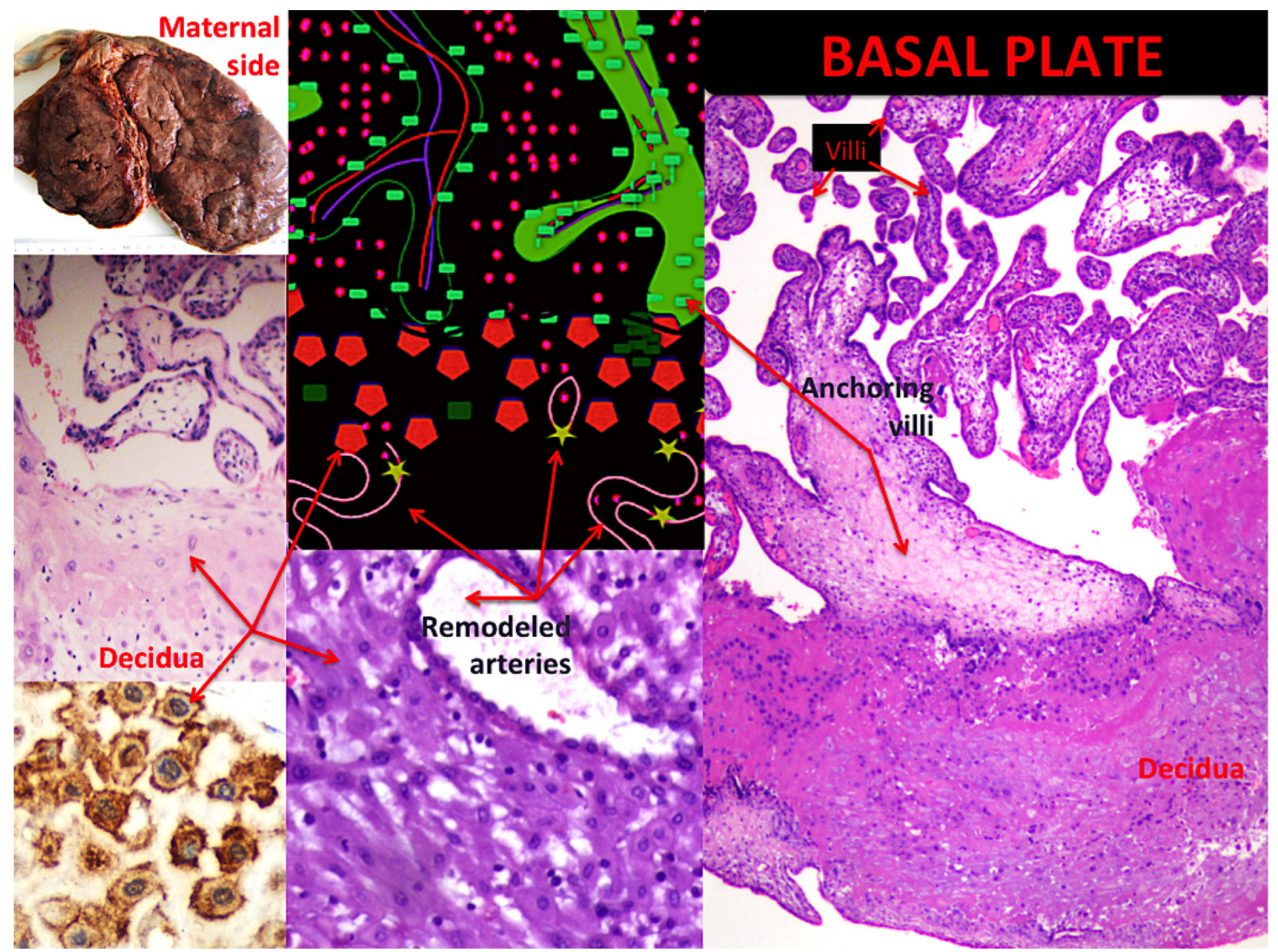

Figure 7. The basal plate 2

A - Gross placental morphology of maternal surface, bilobate shape is recognized. B- The figure represents some contact between anchoring villi and basal plate. Maternal spiral arteries can be seen. C- Decidua cell are pale, with bland nucleus (H\&E 10X). D-The decidua is delineated by immunohistochemistry staining (VEGF-R1 40X). E- A remodeled artery is shown, the once normal thick wall is now, during normal gestation, vena-like (H\&E 10X). F-The anchoring villous is observed in contact with the decidua (H\&E 4X).

There are other special structures, the anchoring villi, which attach the placental tree to the decidualized endometrium, forming columns of trophoblast [Figure 7]. Other specialized septa divide placental regions into cotelydons.

\section{Maternal surface- basal plate}

The above mentioned cotyledons are easily discernible, grossly recognizable circular structures [Figures $6 \mathrm{~A}$ and $7 \mathrm{~A}$ ]. They are inconspicuously covered by decidua, called basal decidua. Microscopically, the basal plate represents the other fetus-maternal interface, where the immunological dialogue occurs. The invasive trophoblast penetrates the decidua at the implantation site and remodels the maternal arterioles, including the endovascular extravillous trophoblast and the interstitial extravillous trophoblast, both kinds of trophoblast work together in vascular remodeling [30] [Figure 6]. Beneath the decidua, the above mentioned insulation trophoblast is also present [Figure $6 \mathrm{C}$ ]. This part of the placenta is currently the most interesting because of its link to gestational diseases. The interaction between histiocytes and lymphocytes has not been determined; we know only that it is important. Large numbers of histiocytes and lymphocytes in the decidua, especially in the presence of plasma cells, suggest pathology. Endovascular extravillous trophoblast, interstitial extravillous trophoblast, columns of trophoblast and anchoring villi play a major role in fetal-placental interaction and correct gestational course, but their different behaviors deserve further study. The decidual component is also important, including spiral arteries; however, their active function in supplying blood remains poorly understood [Figures 6 and 7].

Limitations of the present study are related to population type; selection bias is due to our hospital's highly complex population, which means that a larger number of complicated gestations with abnormal placentas exist than in the number shown; many of these gestations also belong to the Hypertensive Disorders of Pregnancy context. We would like to have access to before-and-after studies in order to better 
compare our students' comprehension of the information they have been provided.

\section{Conclusion}

The placenta is now, more than ever, the focus of multiple investigations related to the evolution, development, health and etiology of disease. New and improved approaches are needed to further our knowledge on the placenta.

\section{Acknowledgements}

We wish to thank the Hospital Universitario San Ignacio- Pontificia Universidad Javeriana for its indispensable support and participation in this study. We also extend our thanks to the parents who participated in this study and to Steven W. Bayless for the English text correction.

\section{Financial Disclosure}

This study makes up part of the research project entitled, "Factors that determine umbilical cord length", [ID PPTA 00005140] financed by the Pontificia Universidad Javeriana and its Hospital Universitario San Ignacio. Bogota, Colombia.

\section{References}

1. Burton GJ, Jauniaux E (2015) What is the placenta? Am J Obstet Gynecol 213: S6. [crossref]

2. Grigsby PL. Animal Models to Study Placental Development and Function throughout Normal and Dysfunctional Human Pregnancy. Semin Reprod Med 34:11-6.

3. Redline RW1 (2015) Classification of placental lesions. Am J Obstet Gynecol 213: S21-28. [crossref]

4. Gillan JE1 (1992) Perinatal placental pathology. Curr Opin Obstet Gynecol 4: 286-294. [crossref]

5. Baergen RN1 (2007) The placenta as witness. Clin Perinatol 34: 393-407. [crossref]

6. Redline RW1 (2008) Placental pathology: a systematic approach with clinical correlations. Placenta 29 Suppl A: S86-91. [crossref]

7. Howat A. Histopathology and cytopathology of limited or no clinical value. Work Party Hystopathology Cytopathol Ltd or No Clin Value 1-13.

8. Redline RW (2013) Correlation of Placental Pathology with Perinatal Brain Injury. Surg Pathol Clin 6: 153-180. [crossref]

9. Redline RW, Minich N, Taylor HG, Hack M (2007) Placental lesions as predictors of cerebral palsy and abnormal neurocognitive function at school age in extremely low birth weight infants. Pediatr Dev Pathol 10:282-92.

10. Gilbert-Barness E, Debich-Spicer D (2005) The placenta. In: Gilbert-Barness E, Debich-Spicer D, editors. Handbook of Pediatric Autopsy Pathology. First. New Jersey: Humana Press 117-42.

11. Boyd T, Redline R. Pathology of the placenta (2007) In: Gilbert-Barness E, editor. Potter's Pathology of the fetus, infant and child. Second. Philadelphia: Mosby Elsevier 645-93.

12. Ahmed A, Ramma W (2015) Unravelling the theories of pre-eclampsia: are the protective pathways the new paradigm? Br J Pharmacol 172: 1574-1586. [crossref]

13. Schlembach D (2003) Pre-eclampsia--still a disease of theories. Fukushima J Med Sci 49: 69-115. [crossref]

14. Mignini LE, Villar J, Khan KS (2006) Mapping the theories of preeclampsia: the need for systematic reviews of mechanisms of the disease. Am J Obstet Gynecol 194: 317-321. [crossref]

15. Maltepe E, Fisher SJ (2015) Placenta: the forgotten organ. Annu Rev Cell Dev Biol 31: 523-552. [crossref]

16. Carter AM (2007) Animal models of human placentation--a review. Placenta 28 Suppl A: S41-47. [crossref]

17. Benirschke K, Burton G, Baergen R. Abortion and the Placenta in Chromosomal Anomalies. In: Benirschke K, Burton G, Baergen R, editors. Pathology of the human placenta. 6th ed. New York: Springer 173-89.

18. Stanek J (2016) Chorangiosis of Chorionic Villi: What Does It Really Mean? Arch Pathol Lab Med 140: 588-593. [crossref]
19. Mayhew TM, Burton GJ (1997) Stereology and its impact on our understanding of human placental functional morphology. Microsc Res Tech 38:195-205.

20. Redline RW. Elevated circulating fetal nucleated red blood cells and placental pathology in term infants who develop cerebral palsy. Hum Pathol 39:1378-84.

21. Pijnenborg R, Vercruysse L, Hanssens M (2006) The uterine spiral arteries in human pregnancy: facts and controversies. Placenta 27:939-58.

22. Mirkovic J, Elias K, Drapkin R, Barletta JA, Quade B, et al. (2015) GATA3 expression in gestational trophoblastic tissues and tumours. Histopathology 67:636-44.

23. Ernst LM (2011) Placenta. In: Ernst LM, Ruchelli ED HD, editor. Color Atlas of Fetal and Neonatal Histology. First edition. Chicago: Springer 363-88.

24. Ferguson VL, Dodson RB (2009) Bioengineering aspects of the umbilical cord. Eur J Obstet Gynecol Reprod Biol 144 Suppl 1: S108-113. [crossref]

25. Baergen R (2005) Microscopic Evaluation of the Second and Third Trimester Placenta. In: Baergen RN, editor. Manual of Benirschke and Kaufmann's Pathology of the Human Placenta. First. New York: Springfield 45-66.

26. Chan JSY, Baergen RN (2012) Gross umbilical cord complications are associated with placental lesions of circulatory stasis and fetal hypoxia. Pediatr Dev Pathol 15:487-94.

27. Lowe JS AP (2014) Reproductive system: placental structure. In: Lowe JS AP, editor. Human Histology: With student consultt Online Access. Third edition. Philadelphia: Elsevier Health Sciences 345-71.

28. Walentin K, Hinze C, Schmidt-Ott KM (2016) The basal chorionic trophoblast cell layer: An emerging coordinator of placenta development. BioEssays 38:254-65.

29. Zeldovich VB, Clausen CH, Bradford E, Fletcher DA, et al. Placental syncytium forms a biophysical barrier against pathogen invasion. PLoS Pathog 9:e1003821.

30. Castellucci M, Kaufmann P (2006) Basic Structure of the Villous Trees. In: Benirschke K, Burton G, Baergen R., editors. Pathology of the human placenta. 6th ed. New York: Springer 50-120

31. Benirschke K, Burton G, Baergen R (2012) Histology of the Chorionic Villi, Fetal Membranes, and Umbilical Cord. In: Benirschke K, Burton G, Baergen R., editors. Pathology of the human placenta. 6th ed. New York: Springer 80-95.

32. Brosens IA, Robertson WB, Dixon HG (1972) The role of the spiral arteries in the pathogenesis of preeclampsia. Obstet Gynecol Annu 1: 177-191. [crossref]

\section{Citation:}

Mercedes Olaya-C and Jorge Andrés Franco(2018) Introductory Teaching Tool Utilizing Immunohistochemistry to Explain the Placenta.Ultrasound Gyn Fetal Med Volume 1(1): 1-8. DOI: 10.31038/UGFM.1000101 